\title{
Serum biomarkers of endothelial glycocalyx injury in canine parvoviral infection
}

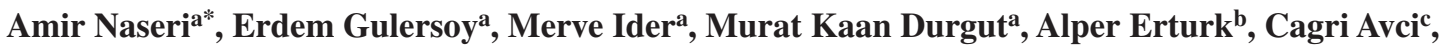 \\ Erman Koral $^{\text {a }}$, Mutlu Sevinc ${ }^{\text {a }}$, Mahmut $\mathbf{O k}^{\mathrm{a}}$
}

\begin{abstract}
Canine parvoviral enteritis (PVE) is one of the most common diseases in young dogs. A range of diseases and inflammatory conditions can cause endothelial glycocalyx (eGCX) disruption, therefore, this study aimed to determine the presence of eGCX damage in dogs with PVE using serum biomarkers of eGCX, and to evaluate their prognostic importance among survivor and non-survivor dogs. Twenty dogs diagnosed with PVE and 10 healthy dogs of both sexes, mixed-breed, and under 6 months of age were included in the study. Clinical examination, blood gas analysis, and complete blood cell counts of the dogs were performed. To detect the eGCX injury, serum endothelial cell-specific molecule-1 (ESM-1), syndecan-1 (SDC-1), angiopoietin-2 (Ang-2), and heparan sulfate (HS) levels were measured. Results showed that at the time of admission serum levels of ESM-1 were higher in dogs with PVE compared to that of the healthy dogs. Dogs with PVE were further assigned into two groups: survivors (n:10) and non-survivors (n:10). The ESM-1 had high sensitivity and specificity to differentiate between survivor and non-survivor dogs with values of $100 \%$ and $67 \%$, respectively, with at an optimum cut-off point of $\geq 460 \mathrm{pg} / \mathrm{mL}$. We concluded that higher levels of ESM-1 in dogs with PVE may indicate eGCX injury when compared to healthy dogs. Also, the high levels of serum ESM-1 in non-survivor dogs suggest that serum ESM-1 may carry some prognostic usefulness for predicting mortality in dogs with PVE.

Key words: vascular endothelium, dog, endothelial cell-specific molecule-1, sepsis, outcome.
\end{abstract}

\section{INTRODUCTION}

The vascular endothelium is the largest organ in the body, forming an interface between the bloodstream and the blood vessel wall. The luminal surface of all vascular endothelial cells is covered by the endothelial glycocalyx (eGCX), which consists of membrane-bound negative charged proteoglycans, glycoproteins, glycolipids, and glycosaminoglycans (Reitsma et al 2007). The main functions of eGCX are maintaining permeability, preventing leukocytes and thrombocyte attachments with endothelium, mechano-transduction, protecting endothelial cells, and reducing inflammatory effects (Hartawan and Wiryana 2019). Previous studies have shown that conditions such as diabetes, chronic kidney disease, hypernatremia, hypervolemia, and ischemic-reperfusion injury can also lead to the eGCX injury (Rehm et al 2001, Nieuwdorp et al 2006, Oberleithner et al 2011, Vlahu et al 2012, Padberg et al 2014). Furthermore, inflammatory conditions such as systemic inflammatory response syndrome and sepsis may also cause eGCX disruption (Henry and

Received: 07.04.2020.

Accepted: 24.07.2020.

aDepartment of Internal Medicine, Faculty of Veterinary Medicine, Selcuk University, Konya, Turkey.

${ }^{b}$ Department of Internal Medicine, Faculty of Veterinary Medicine, Mustafa Kemal University, Hatay, Turkey.

'Department of Virology, Faculty of Veterinary Medicine, Selcuk University, Konya, Turkey.

*Corresponding author: A Naseri; Aleaddin Keykubat Campus, Konya, 42003, Turkey; anaseri@ selcuk.edu.tr
Duling 2000, Nelson et al 2008). Endothelial cell-specific molecule-1 (ESM-1) or endocan, syndecan-1 (SDC-1), angiopoietin-2 (Ang-2), and heparan sulfate (HS) are the most investigated metabolites of eGCX shedding in critically ill patients (Ioakeimidou et al 2017, Uchimido et al 2019).

Canine parvoviral enteritis (PVE) is one of the most important infectious causes of mortality in young puppies (Carmichael 2005). The early lesions consist of necrosis of the crypt epithelial cells in the enteric form of the disease. The villi and lamina propria may collapse completely as a result of the loss of crypt epithelium and the failure of replacing sloughed villous epithelial cells. The loss of digestive epithelium and absorptive surface area presumably results in diarrhoea caused by the combined effect of maldigestion and malabsorption. Death may follow as a result of dehydration, electrolyte imbalance, endotoxic shock, or secondary septicemia (Nandi and Kumar 2010).

Season, purebred nature, bodyweight, vomiting, leukopenia, lymphopenia, thrombocytopenia, hypercoagulability, hypercortisolemia, hypothyroxinemia, hypoalbuminemia, elevated C-reactive protein and tumour necrosis factor, hypocholesterolemia, and hypocitrullinemia have been the preferred variables as diagnostic and prognostic biomarkers in PVE (Schoeman et al 2013). However, there is no literature information about the usage of eGCX in dogs with PVE. Therefore, the present study aimed to determine the presence and magnitude of eGCX damage in dogs with PVE using serum biomarkers of eGCX and to evaluate their prognostic importance to distinguish between the survivor and non-survivor dogs. 


\section{MATERIAL AND METHODS}

ANIMALS

The study protocol was approved by the ethics committee of the Faculty of Veterinary Medicine, Selcuk University (permit number: 2018/140). Twenty dogs suffering from PVE and 10 healthy dogs of both sexes, mixed-breed, and under 6 months of age were included in the study during the years 2018-2019.

All the dogs with PVE had clinical observations of vomiting, anorexia, lethargy, and diarrhoea ranging from mucoid to bloody. Some of the dogs already had sepsis symptoms such as hypothermia, tachycardia, hyperpnea, and severe dehydration at the time of admission. The diagnosis of PVE was confirmed by faecal CPV Ag test (Asan Easy Test PARVO, Asan Pharma. CO. LTD., Gyeonggi-do Korea, sensitivity: $99.5 \%$, specificity: $99.8 \%$ ). None of the dogs enrolled had been vaccinated before the beginning of the study. All the dogs in the PVE group received the same treatment including fluid therapy, antibiotics, and other supportive treatments (antiemetics, vitamin, and amino acid supplementation) during the hospitalisation period. The healthy dogs were chosen among puppies brought to our clinics for routine vaccination and were assigned to the healthy group according to physical examinations, laboratory findings, and negative faecal CPV Ag test results.

\section{SAMPLE COLLECTION}

Five $\mathrm{mL}$ of blood were collected by jugular venipuncture at the time of admission and before discharge from the hospital. One $\mathrm{mL}$ of the collected sample was anaerobically transferred into sodium heparin containing plastic syringes and blood gas analysis was performed immediately. Syringes were prepared by aspirating a small volume of liquid heparin $(5000 \mathrm{IU} / \mathrm{ml})\left(\right.$ Nevparin ${ }^{\circledR}$, Mustafa Nevzat, Turkey) and then expelling it. The heparin layer covering the inside of the syringe prevents the blood sample from coagulation. An extra $\mathrm{mL}$ of the blood was put into the tubes containing $\mathrm{K}_{3}$ EDTA and a complete blood count (CBC) analysis was performed immediately. The remaining $3 \mathrm{~mL}$ of collected blood was put into the tubes without anticoagulant, centrifuged at $2000 \mathrm{x} g$ for $5 \mathrm{~min}$ at $4{ }^{\circ} \mathrm{C}$, and serum samples were extracted. They were stored at $-80{ }^{\circ} \mathrm{C}$ and defrosted immediately before the enzyme-linked immunosorbent assay (ELISA) analysis.

\section{BLOOD GASES AND COMPLETE BLOOD COUNT}

A venous blood gas analysis which included $\mathrm{pH}$, the partial pressure of carbon dioxide $\left(\mathrm{pCO}_{2}\right)$, lactate, sodium $(\mathrm{Na})$, calcium $(\mathrm{Ca})$, chloride $(\mathrm{Cl})$, potassium $(\mathrm{K})$, glucose, base excess (BE), and bicarbonate $\left(\mathrm{HCO}_{3}\right)$ was performed using an automatic blood gas analyser (ABL 90 Flex,
Radiometer, USA). CBCs including total leukocytes, lymphocytes, granulocytes, erythrocytes, mean corpuscular volume (MCV), hematocrit (HCT), mean corpuscular haemoglobin concentration (MCHC), haemoglobin, and platelets were done using an automatic cell counter (MS4e, Melet Schlosing Laboratories, France).

\section{ENDOTHELIAL GLYCOCALYX RELATED BIOMARKERS}

Serum endothelial cell-specific molecule-1 (ESM-1), syndecan-1 (SDC-1), angiopoietin-2 (Ang-2), and heparan sulfate (HS) levels were measured according to the manufacturer's protocol using canine ESM-1 commercial sandwich ELISA kit (MyBioSource, USA, Lot: 20190809C), canine syndecan-1 commercial ELISA kit (MyBioSource, USA, Lot: 08/2019), canine angiopoietin-2 commercial ELISA kit (MyBioSource, USA, Lot: 36343442), and canine heparan sulfate commercial ELISA kit (MyBioSource, USA, 20190809C). Intra-assay coefficients, inter-assay coefficients, and minimum detectable concentrations were $<10 \%,<10 \%$ and $1 \mathrm{pg} / \mathrm{mL}$ for ESM- $1,<15 \%$, $<$ $15 \%$ and $6.25 \mu \mathrm{g} / \mathrm{mL}$ for SDC $-1, \leq 8 \%, \leq 12 \%$ and 0.15 $\mathrm{ng} / \mathrm{mL}$ for Ang- $2,<10 \%,<10 \%$ and $0.1 \mathrm{ng} / \mathrm{mL}$ for HS.

\section{STATISTICAL ANALYSIS}

Data analysis was performed using statistical software (SPSS 25.00). To determine whether the variables had normal distributions, the one-sample KolmogorovSmirnov test was used. Parametric data were evaluated by Student $t$-test while mean \pm standard deviation (SD) and non-parametric data were evaluated by Mann-Whitney U test as median $(\mathrm{min} / \mathrm{max})$. A linear regression analysis was made to determine the independent predictors of mortality. The prognostic values of endothelial related biomarkers were evaluated using receiver operating characteristic (ROC) curve analysis to determine the prognostic cut-off values for the best differentiation between survivors and non-survivors of PVE. Probability curve (survival) was also conducted for serum ESM-1. Statistical significance was considered as $P<0.05$.

\section{RESULTS}

The dogs with PVE presented various weights $(8.92 \pm 4.81$ $\mathrm{kg})$ and ages (3.8 \pm 1.28 months) and the healthy dogs had similar weights $(10 \pm 1.95 \mathrm{~kg})$ and ages ( $3.2 \pm 0.48$ months). There were no statistically significant differences in body weight and age between the groups $(P>0.05)$. Ten dogs in the PVE group survived and were discharged from the hospital 72 hours after the admission and were followed up for the next 14 days by a telephone call to the owners. During the hospitalisation period, 9 dogs in the PVE group died within the first 24 hours and another dog died between 24 and 48 hours. None of the dogs was euthanised during the study. 
Clinical findings showed that dogs with PVE had a higher respiration rate and heart rate $(47.09 \pm 18.9$ and $143.53 \pm 33.65$, respectively) than healthy dogs $(22.13 \pm 6.25$ and 135.60 \pm 6.96 , respectively) $(P<0.05)$. The body temperature did not differ between PVE $(38.05 \pm 1.66)$ and healthy dogs $(38.47 \pm 0.42)(P>0.05)$. The results also revealed that at the time of admission the survivor dogs were not as severely ill as the non-survivor dogs. Higher respiration rate, heart rate, dehydration rate, prolonged capillary refill time (CRT), more variations in consciousness, and haemorrhagic diarrhoea were the most characteristic features in the survivor and non-survivor dogs (table 1).

The blood gases analysis and $\mathrm{CBC}$ results showed no significant differences between healthy dogs and dogs with PVE $(P>0.05)$. Also, there were no significant differences between the time of admission and discharge from the hospital (supplementary table $1^{1}$ ).

The results of serum biomarkers of endothelial glycocalyx showed that levels of ESM-1 were higher in dogs with PVE compared to those of healthy dogs and before discharge (table 2, $P<0.05$ ). There were no significant changes in the levels of SDC-1, Ang-2, and HS between the healthy dogs and dogs with PVE (table 2, P>0.05).

The linear regression analysis showed a significant slope $(\mathrm{F}(1,18)=7.711, P<0.012)$ with an $\mathrm{R}^{2}=0.300$ to predict mortality for dogs with PVE based on their serum ESM-1 levels (table 3, supplementary figure $1^{2}$ ). The same slope was not found for serum levels of
SDC-1, Ang-2, and HS $(P>0.05)$. Also, the results of the PVE group showed that the concentrations of ESM-1 from the non-survivor dogs were significantly higher than those of the survivor dogs $(P<0.05)$ (supplementary figure $2^{3}$ ). Receiver operating characteristic curve (ROC) analysis for the utility of ESM-1, SDC-1, Ang-2 and HS in differentiating between the survivor and non-survivor dogs estimated an area under the curve (AUC) of 0.821 ( $\mathrm{p}=$ $0.022,95 \% \mathrm{CI}=0.615-1.000$ ) for ESM-1 (table 4, figure 1). An optimum cut off point of $460 \mathrm{pg} / \mathrm{mL}$ corresponds to a sensitivity of $100 \%$ and a specificity of $67 \%$ for prediction of mortality (table 4). Survival probability curve for serum endothelial cell-specific molecule-1 (ESM-1) concentrations in dogs with PVE demonstrated that the highest serum concentrations of ESM-1 were associated with the lower prediction of survival in dogs with PVE (figure 2).

\section{DISCUSSION}

Acute enteritis is the most common clinical manifestation of PVE mostly seen in puppies up to 6 months of age. The non-specific clinical signs in the early stages of the disease include depression, anorexia, and lethargy. Vomiting and mucoid to hemorrhagic diarrhoea are the typical signs of the PVE and they develop in the later stages of the disease (Goddard and Leisewitz 2010). Likewise, the puppies in the PVE group of this study had variable signs of anorexia and depression. Also, all of the dogs assigned

Table 1. Clinical parameters in the healthy (n:10), the survivor (n:10) and the non-survivors (n:10) dogs. Body temperature, respiration rate, and heart rate are presented as mean \pm SD. Dehydration rate, capillary refill time, mucosal membranes, mental status, and the type of diarrhea are presented as median and range in parentheses.

\begin{tabular}{lccc}
\hline \multirow{2}{*}{ Parameter } & $\begin{array}{c}\text { Healthy } \\
\text { dogs }\end{array}$ & \multicolumn{2}{c}{ Dogs with PVE at time of admission } \\
\cline { 3 - 4 } & & Survivors & Non-survivors \\
\hline Body temperature $\left({ }^{\circ} \mathrm{C}\right)$ & $38.47 \pm 0.42$ & $38.65 \pm 0.38$ & $38.21 \pm 0.71$ \\
Respiration rate $(\mathrm{bpm})$ & $22.13 \pm 6.25$ & $26.22 \pm 5.39^{\mathrm{a}, \mathrm{b}}$ & $37.12 \pm 3.28^{\mathrm{a}}$ \\
Heart rate (bpm) & $135.60 \pm 6.96$ & $141.11 \pm 18.12^{\mathrm{a}, \mathrm{b}}$ & $158.36 \pm 10.29^{\mathrm{a}}$ \\
Dehydration rate $(\%)$ & $1(1)$ & $2(1-2)^{\mathrm{b}}$ & $3(1-3)^{\mathrm{a}}$ \\
Capillary refill time (sec.) & $1(1)$ & $2(1-2)^{\mathrm{a}, \mathrm{b}}$ & $3(2-3)^{\mathrm{a}}$ \\
Mucous membranes & $2(2)$ & $3(1-4)^{\mathrm{a}}$ & $3(1-4)^{\mathrm{a}}$ \\
Mental status & $4(4)$ & $3(1-4)^{\mathrm{a}, \mathrm{b}}$ & $2(1-3)^{\mathrm{a}}$ \\
Type of diarrhea & $1(1)$ & $2(2-3)^{\mathrm{a}, \mathrm{b}}$ & $3(2-3)^{\mathrm{a}}$ \\
\hline
\end{tabular}

${ }^{a}$ Comparison between healthy dogs $(P<0.05)$.

bComparison between non-survivor dogs $(P<0.05)$.

Dehydration rate: $<5 \%$ (1); 6-8\% (2); 9-10\% (3); $>10 \%$ (4).

Capillary refill time: $<2 \sec (1) ; 3-4 \sec (2) ; 4-5 \sec (3)$.

Mucous membranes: hyperemic (1), normal (2), slightly pale (3), pale (4).

Mental state: comatose (1), depressive (2), alert (3), active (4).

Diarrhoea: none (1), mucoid diarrhoea (2), hemorrhagic diarrhoea (3).

\footnotetext{
Available at www.australjvs.cl/ajvs

2 Available at www.australjvs.cl/ajvs
}

3 Available at www.australjvs.cl/ajvs 
Table 2. Comparison of eGCX related biomarkers in the healthy dogs (n:10), dogs with PVE at admission (n:20), and before discharge (n:10). Data are presented as mean \pm SD and median and range in parentheses.

\begin{tabular}{lccc}
\hline \multirow{2}{*}{ Parameter } & $\begin{array}{c}\text { Healthy } \\
\text { dogs }\end{array}$ & Admission & Dogs with PVE \\
\cline { 2 - 3 } & $283.64 \pm 114.15$ & $644.11 \pm 340.36^{\text {a }}$ & Before discharge \\
\hline ESM-1 $(\mathrm{pg} / \mathrm{mL})$ & $25(12-63)$ & $28(13-168)$ & $313.26 \pm 82.60^{\mathrm{b}}$ \\
SDC-1 $(\mu \mathrm{g} / \mathrm{mL})$ & $9.01 \pm 5.67$ & $8.97 \pm 7.02$ & $35(14-114)$ \\
Ang-2 $(\mathrm{ng} / \mathrm{mL})$ & $5.78 \pm 3.19$ & $7.35 \pm 2.21$ & $9.80 \pm 3.51$ \\
HS $(\mathrm{ng} / \mathrm{mL})$ & & $8.09 \pm 2.72$ \\
\hline
\end{tabular}

${ }^{\mathrm{a} C}$ Comparison between healthy dogs $(P<0.05)$.

${ }^{\mathrm{b}}$ Comparison between time of admission $(P<0.05)$.

ESM-1, endothelial cell-specific molecule-1; SDC-1, syndecan-1; Ang-2, angiopoetin-2; HS, heparan sulfate.

Table 3. Summary of regression analysis of serum biomarkers of eCGX in 10 survivors and 10 non-survivor dogs with $\mathrm{PVE}$ at the time of admission. Data are presented as mean \pm SD.

\begin{tabular}{lccc}
\hline \multirow{2}{*}{ Parameter } & & Dogs with PVE & \\
\cline { 2 - 4 } & Survivors & Non-survivors & 0.012 \\
\hline ESM-1 $(\mathrm{pg} / \mathrm{mL})$ & $313.26 \pm 82.60$ & $580.48 \pm 292.87$ & 0.901 \\
SDC-1 $(\mu \mathrm{g} / \mathrm{mL})$ & $50.04 \pm 36.14$ & $47.68 \pm 46.66$ & 0.199 \\
Ang-2 $(\mathrm{ng} / \mathrm{mL})$ & $9.80 \pm 3.51$ & $7.63 \pm 3.34$ & 0.056 \\
HS $(\mathrm{ng} / \mathrm{mL})$ & $8.09 \pm 2.07$ & $6.13 \pm 1.48$ & \\
\hline
\end{tabular}

ESM-1, endothelial cell-specific molecule-1; SDC-1, syndecan-1; Ang-2, angiopoetin-2; HS, heparan sulfate.

to the PVE group had hemorrhagic diarrhoea either at the time of admission or later during the hospitalisation period.

Previous studies (Otto et al 1997, de Laforcade et al 2003, Prittie 2004, Yilmaz and Senturk 2007) have demonstrated that disruption of the intestinal barrier due

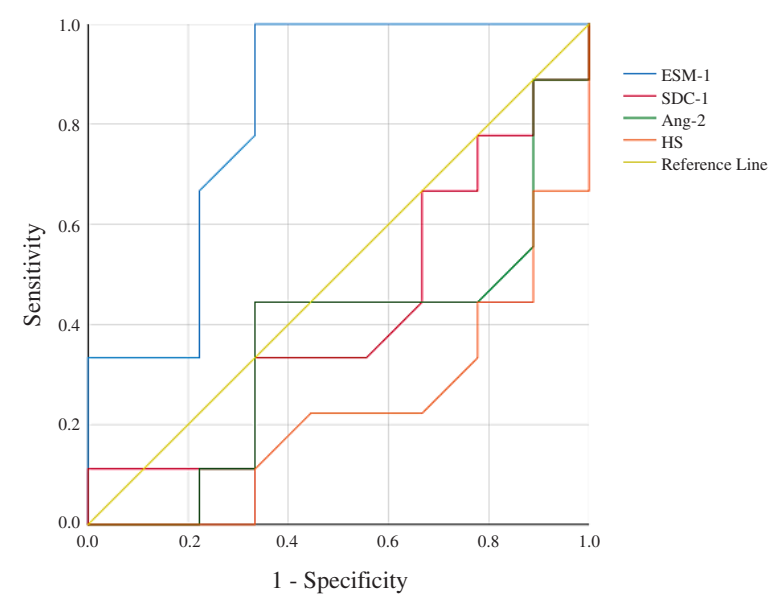

Figure 1. Receiver operating characteristic curve analysis for the differentiation between the survivor and non-survivor dogs with parvoviral enteritis based on the serum endothelial cell-specific molecule-1 (ESM-1), syndecan-1 (SDC-1), angiopoetin-2 (Ang-2) and heparan sulfate (HS) concentration at the time of admission. Results showed that serum ESM-1 (blue line) is the more sensitive and specific biomarker for predicting mortality in the dogs with CPV. to canine parvovirus infection can lead to the translocation of bacteria and endotoxins released from the intestinal tract into the peripheral circulation, with the subsequent development of coliform septicemia. This condition may then lead to SIRS and sepsis. Total leukocyte count $(<6 \times$ $10^{9} / \mathrm{L}$ or $\left.>16 \times 10^{9} / \mathrm{L}\right)$, heart rate $(>160 \mathrm{bpm})$, respiratory rate $(>25$ breaths $/ \mathrm{min})$, and body temperature $\left(<37^{\circ} \mathrm{C}\right.$ or $>39.4{ }^{\circ} \mathrm{C}$ ) are the described criteria for the definition of SIRS and sepsis in dogs (Silverstein and Hoper 2015, Ok et al 2015). These findings were remarkable in the dogs with PVE. The rapid deterioration of the clinical appearance in the non-survivor dogs may be related to the development of SIRS and sepsis.

Studies reporting the mortality rates in the canine PVE have shown that it varies between $25 \%$ and $35 \%$, while it can reach $91 \%$ without treatment (Ling et al 2012). Kocaturk et al (2010) reported a higher mortality rate $(53.4 \%)$ and shorter survival length in the cases of canine PVE. They concluded that insufficient immune system development in younger puppies, sepsis, and related complications were the main causes of death in their study. Our findings indicated that at the time of admission, symptoms of SIRS like tachypnea, tachycardia, hypovolemia, prolonged CRT, and mental status were more serious in the non-survivor dogs compared to the survivors. Also, anamnesis revealed that in the case of non-survivors there were approximately 48 hours between the appearance of the first symptoms (loss of appetite, vomiting, diarrhoea, and stuporous state) and admission to the hospital. As mentioned by Mylonakis 


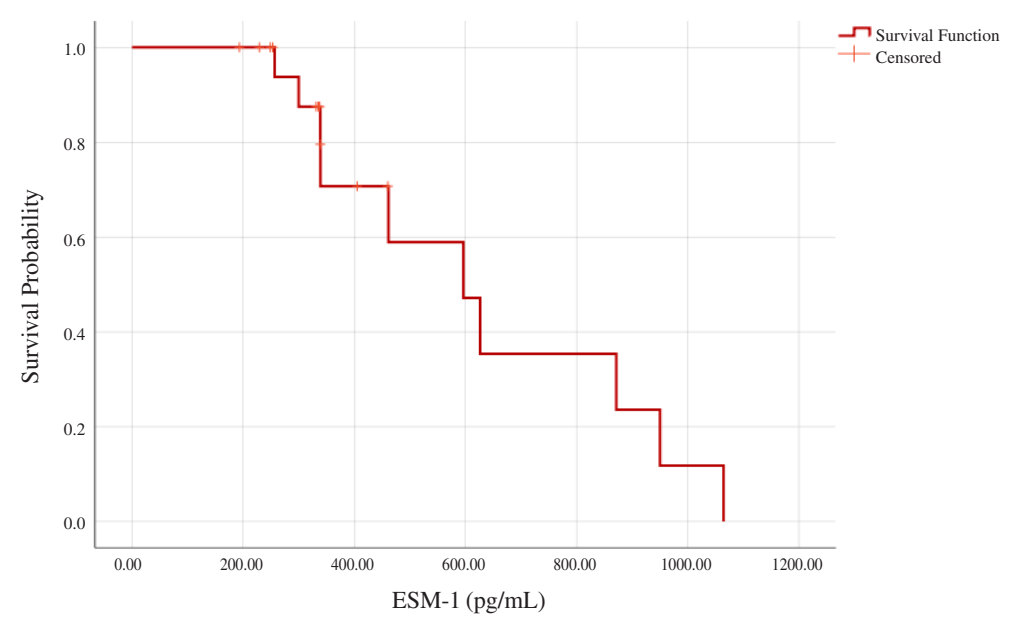

Figure 2. Survival probability curve for serum endothelial cell-specific molecule-1 (ESM-1) concentration in dogs with parvoviral enteritis suggests that the highest serum concentrations of ESM-1 are associated with a lower probability of survival in dogs with PVE.

Table 4. The area under the curve (AUC), standard error, confidence interval (95\%), optimum cut-off values of ESM-1, SDC-1, Ang-2, HS, and respective sensitivity and specificity of mortality prediction in dogs with PVE.

\begin{tabular}{|c|c|c|c|c|c|c|c|c|}
\hline \multirow{2}{*}{ Variable } & \multirow{2}{*}{ AUC } & \multirow{2}{*}{ Standard Error } & \multirow{2}{*}{$P$ value } & \multicolumn{2}{|c|}{$\begin{array}{l}\text { Asymptotic 95\% Confidence } \\
\text { Interval }\end{array}$} & \multirow{2}{*}{ - Cut-off Point } & \multirow{2}{*}{ Sensitivity } & \multirow{2}{*}{ Specificity } \\
\hline & & & & $\begin{array}{c}\text { Lower } \\
\text { Band }\end{array}$ & Upper Bound & & & \\
\hline ESM-1 & 0.821 & 0.105 & 0.022 & 0.615 & 1.000 & 460.92 & 100 & 67 \\
\hline SDC-1 & 0.414 & 0.139 & 0.536 & 0.140 & 0.687 & 41.27 & 66 & 34 \\
\hline Ang-2 & 0.364 & 0.137 & 0.331 & 0.095 & 0.634 & 7.14 & 44 & 67 \\
\hline HS & 0.222 & 0.111 & 0.067 & 0.004 & 0.440 & 7.91 & 44 & 23 \\
\hline
\end{tabular}

ESM-1, endothelial cell-specific molecule-1; SDC-1, syndecan-1; Ang-2, angiopoetin-2; HS, heparan sulfate.

et al (2016), complications such as severe dehydration, hypoperfusion, and the delay in initiation of the treatment probably influenced the survival rates in our study.

Studies in humans with critically ill patients have reported that deterioration of the endothelium of the blood vessels is one of the major complications during systemic inflammation (Cox et al 2015). The vascular endothelium is the largest organ in the body and the endothelial cells line as a single layer along the inner portion of the heart, blood vessels, and lymphatic vessels (Karamysheva 2008). The glycocalyx is a gel-like layer lining the luminal surface of endothelial cells, composed of membrane-bound proteoglycans, glycoproteins, glycosaminoglycans, and adherent plasma proteins (Weinbaum et al 2007).

The eGCX damage can be assessed by different methods. The most common methods to measure eGCX levels in plasma and urine samples in humans are anti-thrombogenic activity assay, electron microscopy, intravital microscopy, side-stream dark-field imaging, and enzyme immunoassay. (Palud et al 2015, Ioakeimidou et al 2017, Iba and Levy 2019). Since there is no available literature data about any standardised methods for eGCX evaluation in dogs, the enzyme immunoassay method (commercial canine ELISA kits) was used in the present study to determine glycocalyx injury in dogs with PVE.

Our results showed that among the serum biomarkers used in the study, concentrations of ESM-1 were higher in dogs with PVE compared to those in the healthy dogs and time before discharge. Other serum biomarkers of eGCX did not show any significant changes between healthy dogs and dogs with PVE. The ESM-1 or endocan is a proteoglycan that is secreted by endothelial cells and the main source of synthesis is the lung endothelial cells (Béchard et al 2001, Seo et al 2015). Experimental studies of human endotoxemia demonstrated that the levels of endocan increased after lipopolysaccharide (LPS) infusion at the beginning of the inflammatory phase and returned to the baseline at $8 \mathrm{~h}$ (Cox et al 2015). A prospective study in septic patients showed that circulating levels of endocan in the septic group were significantly higher than healthy donors and patients with SIRS. They indicated that the concentration of endocan in patients with septic shock 
was prominently higher than that of patients with sepsis and severe sepsis (Scherpereel et al 2006).

Previous studies investigating sepsis demonstrated that pro-inflammatory cytokines such as tumour necrosis factor- $\alpha(\mathrm{TNF}-\alpha)$ and interleukin-1 $\beta$ (IL-1 $\beta)$ as well as bacterial endotoxin and LPS induced the synthesis and release of endocan by endothelial cells. Finally, the sustained hypersecretion of endocan stimulated by LPS and pro-inflammatory cytokines may be in accordance with the high levels of serum endocan in patients with worse outcome (Lassalle et al 1996, Bechard et al 2000). This information suggests that the endocan release could be partly due to endothelial cell injury. Therefore, higher levels of ESM-1 in dogs with PVE compared to healthy dogs migth be related to endothelial cell injury and degradation of eGCX caused by bacterial LPS, endotoxemia, and the release of inflammatory cytokines and endothelial components into the circulation.

Our results showed a significant decrease of ESM-1 levels in the blood samples taken at the time of discharge compared to the levels at the time of admission. In human neonatal septicemia, Zonda et al (2019) found the level of endocan at the time of admission was significantly higher in septic neonates than in non-septic ones and remained high until the $3^{\text {rd }}$ day from admission, before returning to their normal values on day 7 (Lassalle et al 1996, Bechard et al 2000, Zonda et al 2019). The findings of the present study suggest that dogs with PVE had high levels of ESM-1 at the time of admission and after the treatment and the resolution of the inflammatory state, while the levels of ESM-1 in the recovered dogs tended to decrease and were comparable with the healthy group.

The levels of ESM-1 were higher in non-survivor dogs. The analysis of the area under the curve also confirmed that ESM-1 levels at the cut-off point of $460 \mathrm{pg} / \mathrm{mL}$ at the time of admission had prognostic importance for discrimination between the survivor and non-survivor dogs. Scherpereel et al (2006) showed that endocan levels in intensive care unit (ICU) patients were higher in non-survivors than in patients who were still alive 10 days later. Their results suggested that the endocan levels in the blood of septic patients were related to the severity of illness (i.e. higher in septic shock patients when compared to patients with severe sepsis and sepsis). Another study performed by Mihajlovic et al (2014) showed that the concentration of endocan was higher in patients with severe sepsis-induced multi-organ dysfunction in the first $48 \mathrm{~h}$. In their opinion, endothelial damage, which occurs in sepsis, may be a significant factor in the pathogenesis of sepsis-induced organ failure and death. Also, Pauly et al (2016) evaluated the diagnostic and prognostic value of ESM-1 in patients suffering from severe sepsis and septic shock, and found that endocan was able to predict both short- and long-term mortality which was already initiated within the first $24 \mathrm{~h}$ of ICU presentation. Based on our findings, ESM-1 levels were the only prognostic marker of mortality in cases of dogs with PVE. The increased levels of ESM-1 in the non-survivor dogs within the first $24 \mathrm{~h}$ after admission $(9 / 10$ of whole mortality) were due to the severity of clinical findings and the development of sepsis in these dogs. The AUC of 0.821 , a sensitivity of $100 \%$, and a specificity of $67 \%$ suggest that the ESM-1 may carry some prognostic usefulness for predicting mortality in dogs with PVE.

The present study had the following limitations: 1) the study population was relatively small and a re-evaluation of the hypothesis of eGCX injury in larger sample populations is needed, 2) a lack of more frequent blood measurements especially at $24^{\text {th }}$ and $48^{\text {th }}$ hours 3 ) a histopathological examination was not performed in the non-survivor dogs, 4) since the markers of organ dysfunction were not evaluated in the present study, we could not make an association between eGCX injury and organ dysfunction, especially in the non-survivor dogs. As a conclusion, we observed that the levels of ESM-1 were higher in dogs with PVE when compared to healthy dogs. Also, the concentrations of ESM-1 were higher in the non-survivors dogs than in the survivors. At the time of admission, the cut-off point of $\geq 460 \mathrm{pg} / \mathrm{mL}$ for ESM-1 with sensitivity and specificity of $100 \%$ and $67 \%$, respectively, had prognostic importance for discrimination between the survivor and non-survivor dogs with canine PVE. Endothelial specific molecule-1 (ESM-1) is considered to be a promising prognostic biomarker in dogs with PVE.

\section{ACKNOWLEDGEMENTS}

This study was financially supported by the Selcuk University Scientific Research Office (Project no: 19401010).

\section{DECLARATION OF COMPETING INTEREST}

The authors declare no conflict of interest.

\section{REFERENCES}

Béchard D, Gentina T, Delehedde M, Scherpereel A, Lyon M, et al. 2001. Endocan is a novel chondroitin sulfate/dermatan sulfate proteoglycan that promotes hepatocyte growth factor/scatter factor mitogenic activity. J Biol Chem 276, 48341-48349.

Bechard D, Meignin V, Scherpereel A, Oudin S, Kervoaze G, et al. 2000. Characterization of the secreted form of endothelial-cell-specific molecule 1 by specific monoclonal antibodies. J Vasc Res 37, 417-425.

Carmichael LE. 2005. An annotated historical account of canine parvovirus. J Vet Med 52, 303-11.

Cox LA, van Eijk LT, Ramakers BP, Dorresteijn MJ, Gerretsen J, et al. 2015. Inflammation-induced increases in plasma endocan levels are associated with endothelial dysfunction in humans in vivo. Shock 43, 322-326.

de Laforcade AM, Freeman LM, Shaw SP, Brooks MB, Rozanski EA, et al. 2003. Hemostatic changes in dogs with naturally occurring sepsis. J Vet Med 17, 674-679.

Goddard A, Leisewitz AL. 2010. Canine parvovirus. Vet Clin North Am Small Anim Pract 40, 1041-1053.

Hartawan INB, Wiryana M. 2019. The role of endothelial glycocalyx in sepsis. Bali J Anesthesiol 3, 27-32. 
Henry CB, Duling BR. 2000. TNF- $\alpha$ increases entry of macromolecules into luminal endothelial cell glycocalyx. Am J Physiol Heart Circ Physiol 279, 2815-2823.

Iba T, Levy JH. 2019. Derangement of the endothelial glycocalyx in sepsis. J Thromb Haemost 17, 283-294.

Ioakeimidou A, Pagalou E, Kontogiorgi M, Antoniadou E, Kaziani K, et al. 2017. Increase of circulating endocan over sepsis follow-up is associated with progression into organ dysfunction. Eur J Clin Microbiol Infect Dis 36, 1749-1756.

Karamysheva AF. 2008. Mechanisms of angiogenesis. Biochem (Mosc) $73,751-762$.

Kocaturk M, Martinez S, Eralp O, Tvarijonaviciute A, Ceron J, et al. 2010. Prognostic value of serum acute-phase proteins in dogs with parvoviral enteritis. J Small Anim Pract 51, 478-483.

Lassalle P, Molet S, Janin A, Van der Heyden J, Tavernier J, et al. 1996. ESM-1 is a novel human endothelial cell-specific molecule expressed in lung and regulated by cytokines. J Biol Chem 271, 20458-2064.

Ling M, Norris JM, Kelman M, Ward MP. 2012. Risk factors for death from canine parvoviral-related disease in Australia. Vet Microbiol $158,280-290$

Mihajlovic DM, Lendak DF, Brkic SV, Draskovic BG, Mitic GP, et al. 2014. Endocan is useful biomarker of survival and severity in sepsis. Microvasc Res 93, 92-97.

Mylonakis ME, Kalli I, Rallis TS. 2016. Canine parvoviral enteritis: an update on the clinical diagnosis, treatment, and prevention. Vet Med Res Rep 7, 91.

Nandi S, Kumar M. 2010. Canine parvovirus: current perspective. Indian $J$ Virol 21, 31-44.

Nelson A, Berkestedt I, Schmidtchen A, Ljunggren L, Bodelsson M. 2008. Increased levels of glycosaminoglycans during septic shock: relation to mortality and the antibacterial actions of plasma. Shock 30, 623-627.

Nieuwdorp M, Mooij HL, Kroon J, Atasever B, Spaan JA, et al. 2006. Endothelial glycocalyx damage coincides with microalbuminuria in type 1 diabetes. Diabetes 55, 1127-1132.

Oberleithner H, Peters W, Kusche-Vihrog K, Korte S, Schillers H, et al. 2011. Salt overload damages the glycocalyx sodium barrier of vascular endothelium. Pflugers Arch 462, 519.

Ok M, Er C, Yıldız R, Çöl R, Aydoğdu U, et al. 2015. Evaluation of acute phase proteins, some cytokines and hemostatic parameters in dogs with sepsis. Kafkas Univ Vet Fak Derg 21, 761-766.

Otto CM, Drobatz KJ, Soter C. 1997. Endotoxemia and tumor necrosis factor activity in dogs with naturally occurring parvoviral enteritis. $J$ Vet Med 11, 65-70.
Padberg JS, Wiesinger A, di Marco GS, Reuter S, Grabner A, et al. 2014. Damage of the endothelial glycocalyx in chronic kidney disease. Atherosclerosis 234, 335-343.

Palud A, Parmentier-Decrucq E, Pastre J, Caires NDF, Lassalle P, et al. 2015. Evaluation of endothelial biomarkers as predictors of organ failures in septic shock patients. Cytokine 73, 213-218.

Pauly D, Hamed S, Behnes M, Lepiorz D, Lang S, et al. 2016. Endothelial cell-specific molecule-1/endocan: diagnostic and prognostic value in patients suffering from severe sepsis and septic shock. $J$ Crit Care 31, 68-75.

Prittie J. 2004. Canine parvoviral enteritis: a review of diagnosis, management, and prevention. J Vet Emerg Crit Care 14, 167-176.

Rehm M, Haller M, Orth V, Kreimeier U, Jacob M, et al. 2001. Changes in blood volume and hematocrit during acute preoperative volume loading with $5 \%$ albumin or $6 \%$ hetastarch solutions in patients before radical hysterectomy. Anesthesiology 95, 849-856.

Reitsma S, Slaaf DW, Vink H, Van Zandvoort MA, Oude Egbrink MG. 2007. The endothelial glycocalyx: composition, functions, and visualization. Pflugers Arch 454, 345-359.

Scherpereel A, Depontieu F, Grigoriu B, Cavestri B, Tsicopoulos A, et al. 2006. Endocan, a new endothelial marker in human sepsis. Crit Care Med 34, 532-537.

Schoeman JP, Goddard A, Leisewitz AL. 2013. Biomarkers in canine parvovirus enteritis. $N Z$ Vet $J 61,217-222$.

Seo K, Kitazawa T, Yoshino Y, Koga I, Ota Y. 2015. Characteristics of serum endocan levels in infection. PLoS One 10, e0123358.

Silverstein DC, Hoper K. 2015. Small Animal Critical Care Medicine. $2^{\text {nd }}$ ed. Elsevier Saunders, St. Louis, USA.

Uchimido R, Schmidt EP, Shapiro NI. 2019. The glycocalyx: a novel diagnostic and therapeutic target in sepsis. Critical Care 23, 16.

Vlahu CA, Lemkes BA, Struijk DG, Koopman MG, Krediet RT, et al. 2012. Damage of the endothelial glycocalyx in dialysis patients. Clin J Am Soc Nephrol 23, 1900-1908.

Weinbaum S, Tarbell JM, Damiano ER. 2007. The structure and function of the endothelial glycocalyx layer. Annu Rev Biomed Eng 9, 121-167.

Yilmaz Z, Senturk S. 2007. Characterisation of lipid profiles in dogs with parvoviral enteritis. J Small Anim Pract 48, 643-650.

Zonda GI, Zonda R, Cernomaz AT, Paduraru L, Avasiloaiei AL, et al. 2019. Endocan-a potential diagnostic marker for early onset sepsis in neonates. J Infect Dev Ctries 13, 311-317. 
